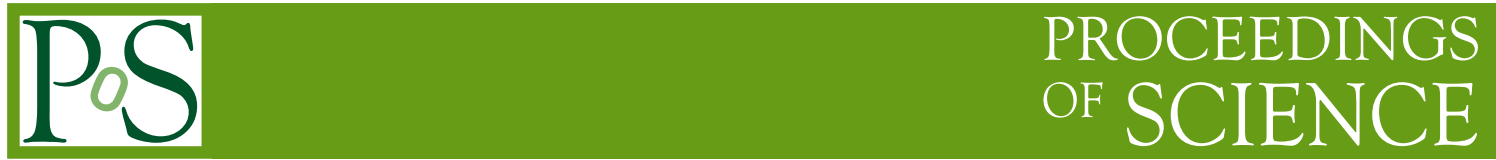

\title{
A Highly Polarised Jet in XTE J1748-288
}

\author{
Catherine Brocksopp* \\ Mullard Space Science Laboratory, University College London, UK \\ E-mail: cb4@mssl.ucl.ac.uk
}

\section{Rob Fender}

University of Southampton, $U K$

E-mail: rpf@phys.soton.ac.uk

\section{James Miller-Jones}

University of Amsterdam, The Netherlands

E-mail: jmillerescience.uva.nl

\section{Ben Stappers}

Stichting ASTRON, The Netherlands

E-mail: stapperseastron.nl

XTE J1748-288 is a black hole X-ray transient which went into outburst in 1998 June. The $\mathrm{X}$-ray lightcurves showed fairly canonical morphologies with minor variations on the "Fast Rise Exponential Decay" profile. The radio source, however, reached an unusually high flux density of over $600 \mathrm{mJy}$. This high radio flux was accompanied by high ( $>20 \%)$ fractional linear polarisation, the variability of which was anti-correlated with the flux density. We use this variability to discuss possible depolarisation mechanisms, to predict the underlying behaviour of the core/jet components and to study polarisation properties within the context of internal shock models for jets.

VI Microquasar Workshop: Microquasars and Beyond September 18-22, 2006

Como, Italy

\footnotetext{
${ }^{*}$ Speaker.
} 


\section{Introduction}

The X-ray outburst of XTE J1748-288 began on 1998 June 3-4 when it was detected by the All Sky Monitor (ASM) and Burst and Transient Source Explorer (BATSE) instruments on-board $R X T E$ and $C G R O$ respectively. The source was initially hard but beginning to soften at BATSE energies (see Brocksopp et al. 2006 and references therein). A later, more detailed analysis of the X-ray data suggested that the outburst started in the very high state (VHS) with an unusually dominant power-law component (Revnivtsev et al. 2000). As the X-ray source decayed following the outburst, it passed through the high/soft state (HSS) to the low/hard state (LHS), in a manner which is now considered "canonical" behaviour (e.g. Fender et al. 2004).

An optically-thin, variable radio source was detected 1998 June 7 and was later resolved on June 14-15 (Hjellming et al. 1998a, b). XTE J1748-288 became the third known Galactic radio source to display apparent superluminal motion and the first to show strong deceleration of the jet in the interstellar medium.

\section{Observations}

XTE J1748-288 was observed at two or four radio frequencies on seven occasions by the Australia Telescope Compact Array (ATCA). It was detected, although unresolved, at each epoch and was found to have unusually high radio flux densities, peaking at $>600 \mathrm{mJy}$. There was also an unusually high level of linear polarisation (LP) with a maximum value of LP/(Stokes I) 23\%.

For comparison with the ATCA data, we have also obtained archival radio data from the Very Large Array (VLA) and Green Bank Interferometer (GBI). We note that there appears to be a discrepancy in flux calibration between the GBI and ATCA/VLA. We therefore ignore the absolute flux densities of the GBI data but tentatively trust the variability. We also use public X-ray data from the RXTE/ASM and CGRO/BATSE archives.

For full details of the observations and data reduction we refer the reader to Brocksopp et al. (2006).

\section{X-ray and Radio Lightcurves}

The X-ray and radio lightcurves are plotted in Fig. 1. Vertical dotted lines indicate the ranges of dates between which the state transitions are thought to have occurred (using Revnivtsev et al. 2000).

The top panel shows the few available datapoints obtained by BATSE in the $20-100 \mathrm{keV}$ range. There appears to be some temporary increase superimposed on the decay which may be related to variability in the GBI lightcurve which we discuss later. This feature does not appear to have a significant counterpart in the second panel, the ASM lightcurve (with energy range 2-12 keV). This ASM plot shows a fairly canonical morphology with only a pair of peaks to distinguish it from a simple "Fast Rise Exponential Decay" profile. Of these two peaks, the second appears to coincide with the transition from VHS to HSS. The first peak appears to take place well within the VHS and is thus dominated by the power-law emission as discussed by Revnivtsev et al. (2000). 


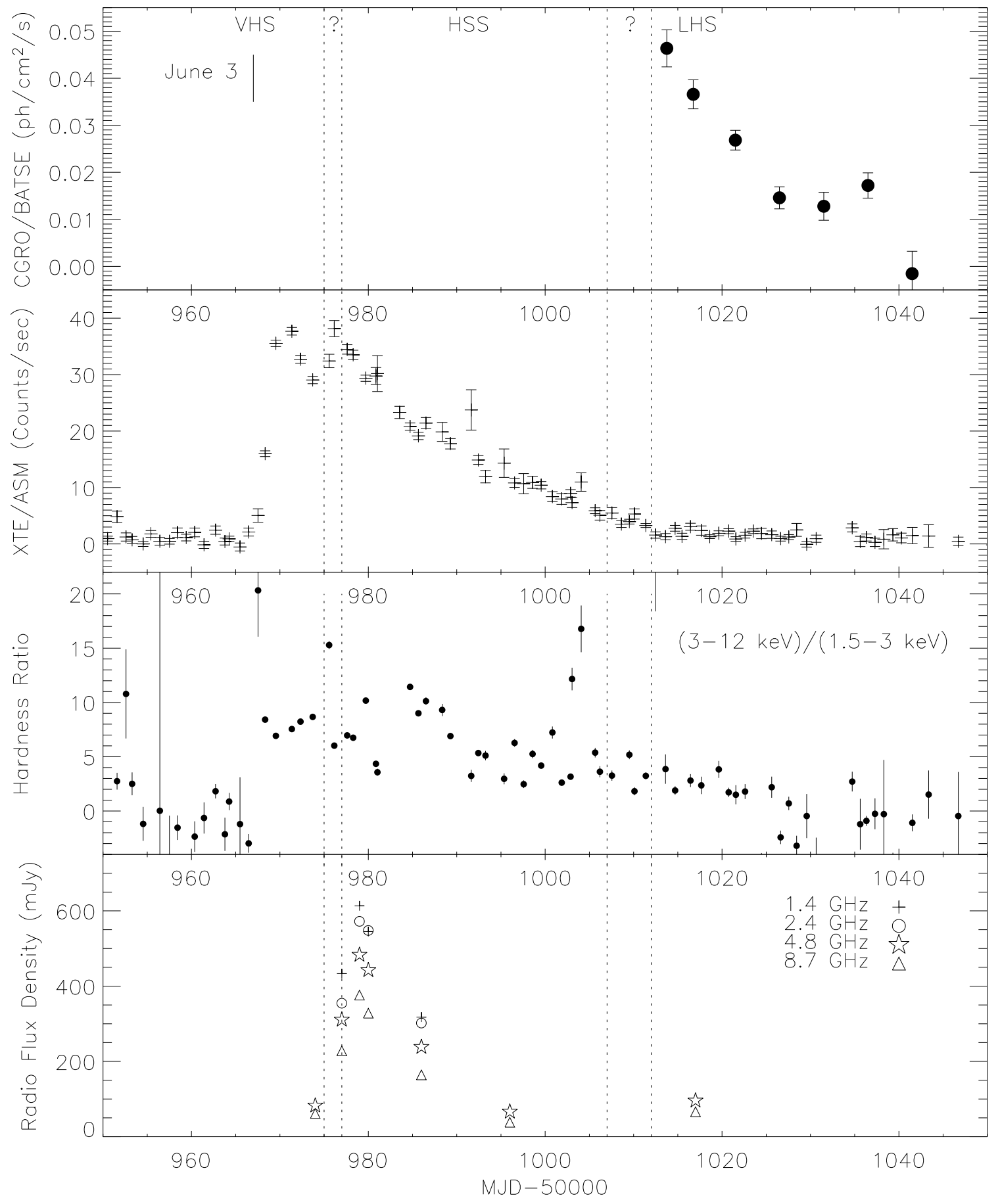

Figure 1: X-ray and radio lightcurves of the 1998 outburst of XTE J1748-288. Top: CGRO/BATSE (20$100 \mathrm{keV})$. Second: RXTE/ASM (1.5-12 keV). Third: RXTE/ASM hardness ratio (3-12 keV)/(1.5-3 keV). Bottom: radio lightcurve from ATCA (with error bars smaller than the symbols). The two pairs of vertical dotted lines indicate the time-ranges during which the transitions from the very high (VHS) to high/soft (HSS) and high/soft to low/hard (LHS) X-ray spectral states are thought to have occurred (using Revnivtsev et al. 2000). The start date of the outburst, June 3 (MJD 30967), is indicated. 
The third panel shows the ASM hardness ratio in the 3-12 keV / 1.5-3 keV range - the X-ray emission is relatively hard, particularly during the VHS, and softens during the decay.

Finally the ATCA data are shown in the bottom panel. The radio outburst appeared to start coincidentally with, or slightly after, the first X-ray peak and just prior to the transition to the HSS. The radio peak and subsequent decay continued during the HSS and into the LHS, although were uncorrelated with the X-ray data. The peak of the outburst reached an extremely high level of 613 mJy at $1.4 \mathrm{GHz}$ and, even once the LHS was reached, remained unusually bright.

\section{Radio Polarisation}

The ATCA radio data are plotted again in the top panel of Fig. 2 on an expanded time axis. The fourth panel shows the spectral index, calculated for all four frequencies where available and also for the 4.8-8.7 GHz data at all epochs. The radio emission remains optically thin throughout our observations, suggesting that even during the LHS, when we would expect to observe a partially self-absorbed jet, the radio flux remains dominated by "contaminating" optically thin emission. The second panel shows the LP lightcurve and the third panel the fractional LP (FP) lightcurve. It is instantly noticeable that the LP flux is correlated, and the FP anti-correlated, with the Stokes I data. The FP is also extremely high $(\sim 23 \%)$ compared with that of other Galactic black hole systems, most of which have been detected at just a few percent, if at all.

Any model invoked to describe the radio emission during transient events needs to be able to explain this anti-correlation with the FP. We first consider possible mechanisms which could produce the observed variability in FP. According to e.g. Longair (1994), the maximum possible FP of an optically thin synchrotron source is $\sim 70 \%$ and of an optically thick synchrotron source is $\sim 10-15 \%$. Reductions in these percentages can come about via wavelength-dependent or wavelength-independent mechanisms. The bottom panel of Fig. 2 shows the observed position angle (PA) of the polarised emission. For the four epochs for which we have data at four frequencies, we use the values of $\mathrm{PA}$, according to $P A=P A_{0}+R M \lambda^{2}$ (where $P A_{0}$ is the intrinsic position angle of the polarised emission and $R M$ is the rotation measure), in order to determine the rotation measure for any Faraday rotation taking place along the line of sight towards XTE J1748-288. We find that $R M$ is consistent with zero, thus suggesting that Faraday rotation is unlikely to make a significant contribution to the observed variability in FP.

Instead we consider wavelength-independent mechanisms for the observed depolarisation. Two possibilities are (i) competing contributions from a polarised jet and depolarised core, as observed in e.g. GRS 1915+105 (Fender et al. 1999) and (ii) multiple "packets", each with a different PA contributing to an observed depolarised "average", e.g. knots within the precessing jets of SS433 (Stirling et al. 2004). With no evidence for a precessing jet, we consider the former option in the next section.

\section{Discussion and Comparison with VLA and GBI data}

In order to test the suggestion that the variability in FP was due to the changing relative contributions of a depolarised core and polarised jet we have obtained quasi-simultaneous VLA archival data, in which the radio source could be resolved into two components aligned east-west. Fig. 3 


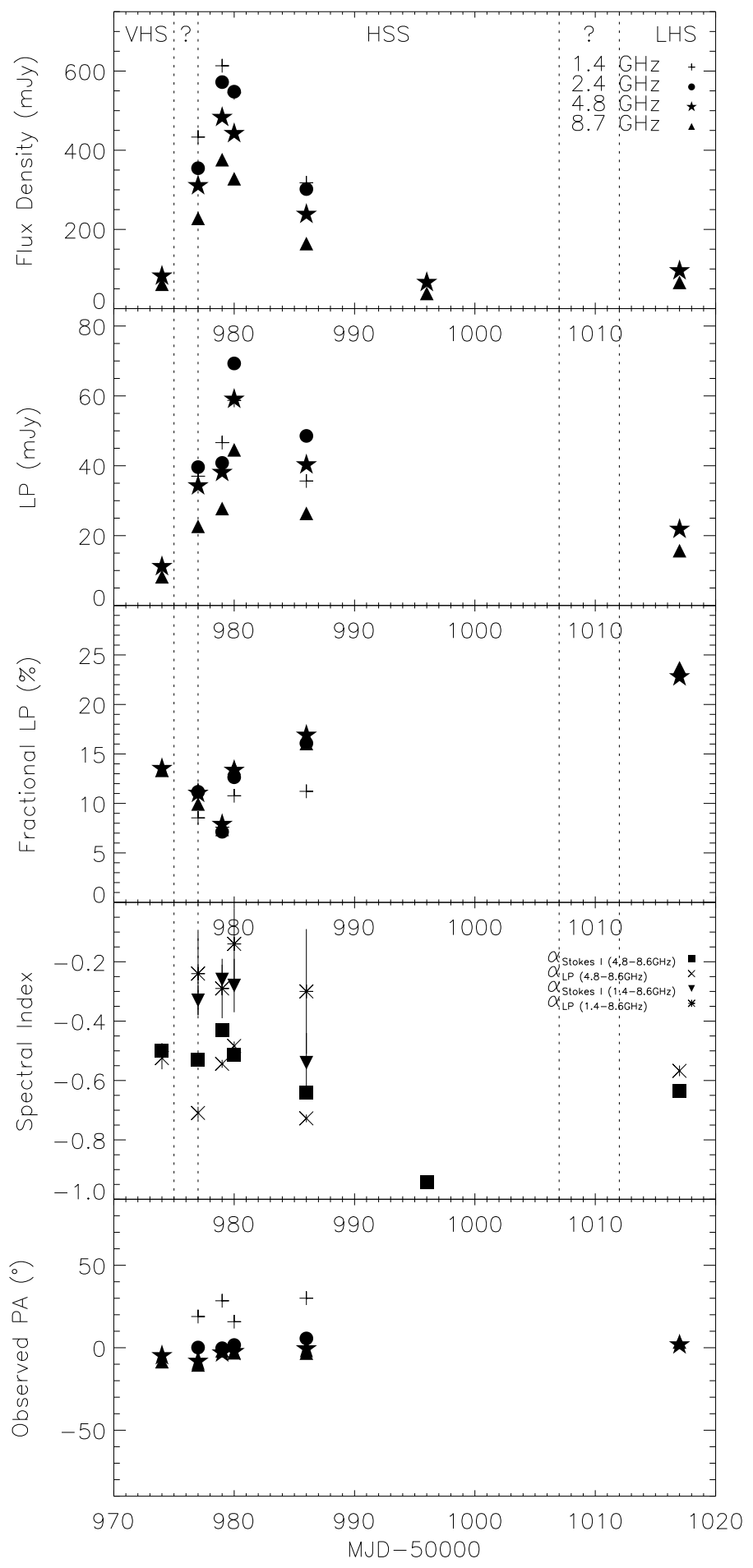

Figure 2: Five plots showing the radio data on an expanded time axis. Top: Stokes $I$ lightcurve. Second: Linear Polarisation lightcurve $\left(\sqrt{Q^{2}+U^{2}}\right)$. Third: Fractional polarisation $\left(100 \times \sqrt{Q^{2}+U^{2}} / I\right)$ lightcurve. Fourth: Spectral Index (obtained using both two and four frequencies and using both Stokes I and LP datapoints). Bottom: Observed polarisation position angle. NB error bars are typically smaller than the symbols. 


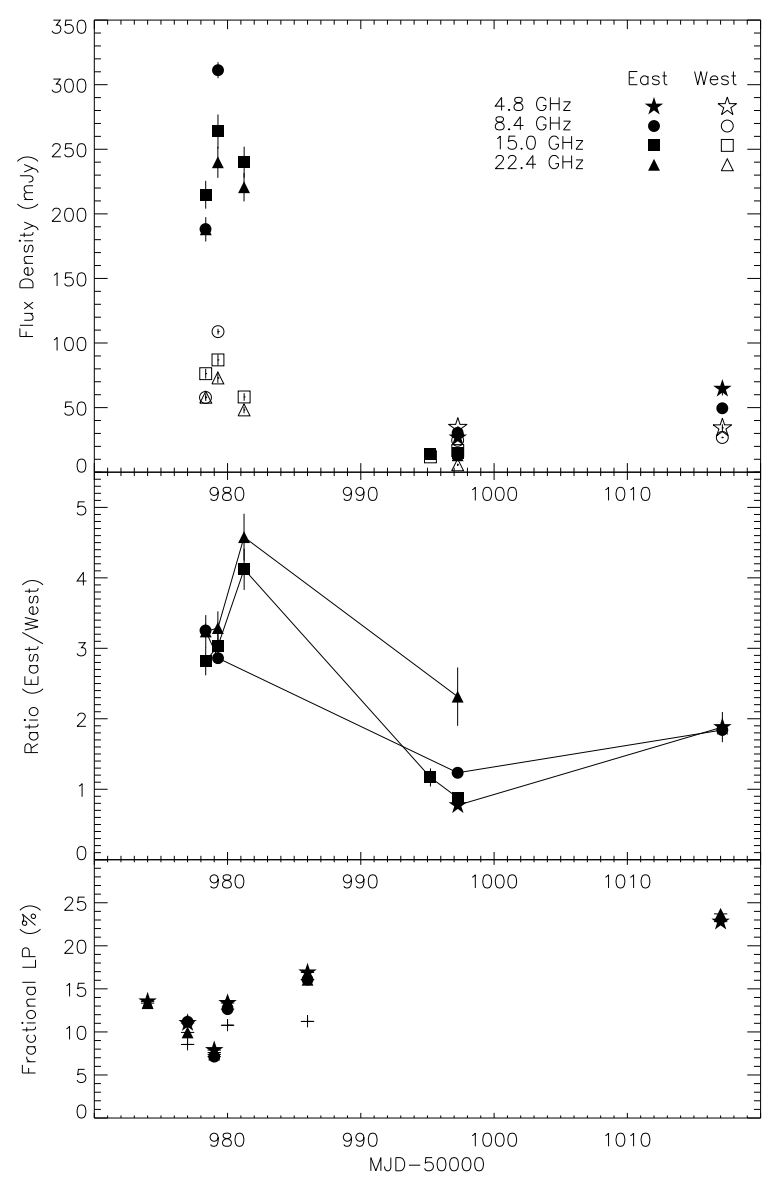

Figure 3: Top: Radio lightcurves from the VLA, using data from epochs most contemporaneous with the ATCA data. We plot separately the contributions from the east (filled symbols) and west (open symbols) components resolved in the images. Middle: Ratio plot of the east:west contributions. Bottom: The FP lightcurve, copied from Fig. 2 for comparison. The eastern component dominates the emission but its relative contribution decreases during the epochs when the ATCA data shows the highest fractional polarisation. This is consistent with our suggestion that the eastern component is the depolarised core and the western component the linearly polarised jet.

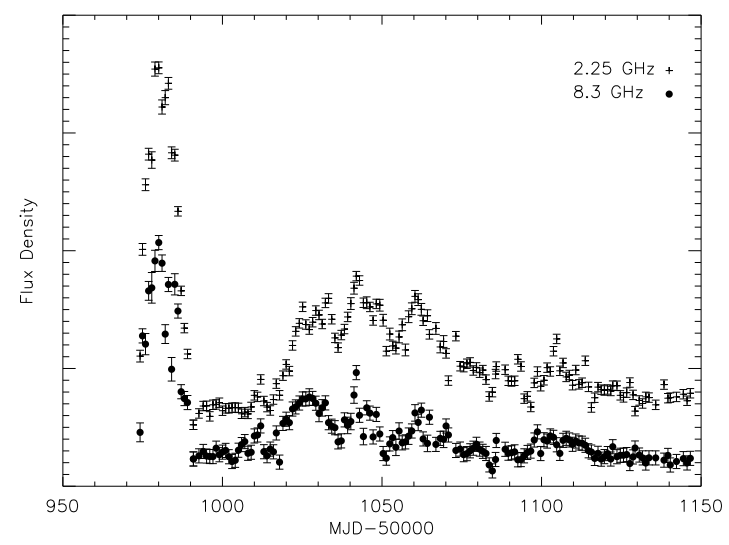

Figure 4: GBI lightcurves showing the peculiar variability of the final low/hard state. We do not label the flux density axis due to the discrepancy in calibration bewteen the GBI and the ATCA/VLA. 
shows that the relative contribution of the eastern component reduced dramatically as the total radio emission decayed and the FP increased, suggesting that the eastern component could represent the core and the western component the jet. This scenario is complicated, however, by the high FP prior to the radio peak - the FP and Stokes I flux density (e.g. Fender et al. 2004) are too high to be residual LHS emission so was there an additional, earlier optically-thin ejection, and hence polarised jet component?

Alternatively if we consider the internal shock model of e.g. Fender et al. (2004), the peak of the emission should correspond to the collision between the fast jet and the earlier, slower jet of the LHS. This collision then coincides with the dip in FP, perhaps suggesting that the collision causes a temporary disruption in magnetic field before it is re-ordered by the shock.

Finally we need to address the high flux density and FP once the X-ray source has returned to the LHS. The observed flux density rises to 50-100 mJy at this time, unprecedented behaviour for a "normal" LHS jet. In Fig. 4 we plot archival GBI lightcurves for comparison with the ATCA data. As stated earlier, we do not trust the absolute fluxes but tentatively consider the striking and extremely unusual variability. During the X-ray LHS, the radio flux rises to a bright plateau, superimposed by multiple peaks. It is not clear whether this is the result of a collision with the ISM, the collision of additional internal shocks or additional jet ejections at the HSS-LHS transition (in contravention of Fender et al. 2004).

For a more complete analysis and discussion, please see Brocksopp et al. (2006) as well as future work in which we will perform a more complete analysis of the VLA data.

\section{References}

[1] C. Brocksopp, R.P. Fender, J. Miller-Jones, B. Stappers, A highly polarised radio jet during the 1998 outburst of the black hole transient XTE J1748-288, 2006, MNRAS submitted

[2] R.P. Fender, S.T. Garrington, D.J. McKay, T.W.B. Muxlow, G.G. Pooley, R.E. Spencer, A.M. Stirling, E.B. Waltman, MERLIN observations of relativistic ejections from GRS 1915+105, 1999, MNRAS, 304,865

[3] R.P. Fender, T. Belloni, E. Gallo, Towards a unified model for black hole X-ray binary jets, 2004, MNRAS, 355, 1105

[4] R.M. Hjellming, M.P. Rupen, A.J. Mioduszewski, XTE J1748-288, 1998a, IAUC 6934

[5] R.M. Hjellming, M.P. Rupen, A.J. Mioduszewski, D.A. Smith, B.A. Harmon, E.B. Waltman, F.D. Ghigo, G.G. Pooley, Radio and X-ray Observations of the New Relativistic Jet X-ray Transient XTE J1748-288, 1998b, BAAS, 193, 103.08

[6] M.S. Longair, 1994, High Energy Astrophysics, Vol. 2, Cambridge University Press, Cambridge

[7] M.G. Revnivtsev, S.P Trudolyubov, K.N. Borozdin, RXTE observations of Galactic microquasar XTE J1748-288 during its 1998 outburst, 2000, 312, 151

[8] A.M. Stirling, R.E. Spencer, T.V. Cawthorne, Z. Paragi, Polarization and kinematic studies of SS 433 indicate a continuous and decelerating jet, 2004, MNRAS, 354, 1239 